\title{
P03-8-1 Poster session
}

\section{Sphingomyelin disrupts vesicular trafficking of cholesterol in Niemann-Pick disease type $C$}

\author{
Hiroyuki Nakamura, Masahiro Wanikawa, Shunsuke Emori, Naohiro Hashimoto, \\ Toshihiko Murayama
}

Chiba University, Japan

Niemann-Pick disease type C (NPC) is a neurodegenerative lipid storage disorder caused by mutations in the NPC1 or NPC2 gene. Loss of function of either protein results in the accumulation of cholesterol in late endosomes/lysosomes. NPC1 protein is a key molecule in cholesterol trafficking from late endosome to the Golgi apparatus and the endoplasmic reticulum. In NPC cells, SM is also accumulated in late endosomes/lysosomes, the same as cholesterol. In the present study, we investigated the effect of SM on the intracellular accumulation of cholesterol in NPC cells. Treatment of NPC cells with HPA-12, an inhibitor of the ceramide transfer protein CERT, significantly decreased SM level. In this condition, the accumulation of cholesterol in NPC cells was decreased, which was rescued by supplementation of the culture medium with SM. The accumulation of SM in NPC cells inhibited the transport of LDL-derived free cholesterol to the endoplasmic reticulum. Rab9, a small GTP-binding protein, is a key regulator of intracellular vesicular transport from late endosomes to the Golgi. Overexpression of Rab9 in NPC cells reduced the accumulation of free cholesterol, which was rescued by supplementation of the culture medium with SM. While in NPC cells overexpressed the Rab9 constitutively active mutant, Rab9Q66L, treatment with SM did not lead to the accumulation of free cholesterol. These results suggest that accumulation of SM negatively regulate the NPC1-independent and Rab9-dependent vesicular trafficking in NPC cells, and reduction in SM level in NPC cells recovered the Rab9-dependent vesicular trafficking defect. 